\title{
Developments on spectral characterizations of graphs
}

van Dam, E.R.; Haemers, W.H.

Published in:

Discrete Mathematics

Publication date:

2009

Link to publication in Tilburg University Research Portal

Citation for published version (APA):

van Dam, E. R., \& Haemers, W. H. (2009). Developments on spectral characterizations of graphs. Discrete Mathematics, 309(3), 576-586.

\section{General rights}

Copyright and moral rights for the publications made accessible in the public portal are retained by the authors and/or other copyright own and it is a condition of accessing publications that users recognise and abide by the legal requirements associated with these rights.

- Users may download and print one copy of any publication from the public portal for the purpose of private study or research.

- You may not further distribute the material or use it for any profit-making activity or commercial gain

- You may freely distribute the URL identifying the publication in the public portal

Take down policy

If you believe that this document breaches copyright please contact us providing details, and we will remove access to the work immediate and investigate your claim. 


\title{
Developments on spectral characterizations of graphs
}

\author{
Edwin R. van Dam, Willem H. Haemers* \\ Tilburg University, Department of Econometrics and O.R., P.O. Box 90153, 5000 LE Tilburg, The Netherlands
}

\section{A R T I C L E I N F O}

\section{Article history:}

Received 10 May 2007

Accepted 23 July 2007

Available online 21 September 2008

\section{Keywords:}

Spectra of graphs

Cospectral graphs

Generalized adjacency matrices

Distance-regular graphs

\begin{abstract}
A B S T R A C T
In [E.R. van Dam, W.H. Haemers, Which graphs are determined by their spectrum? Linear Algebra Appl. 373 (2003), 241-272] we gave a survey of answers to the question of which graphs are determined by the spectrum of some matrix associated to the graph. In particular, the usual adjacency matrix and the Laplacian matrix were addressed. Furthermore, we formulated some research questions on the topic. In the meantime, some of these questions have been (partially) answered. In the present paper we give a survey of these and other developments.
\end{abstract}

(C) 2008 Elsevier B.V. All rights reserved.

\section{Introduction}

Since [13] was published, the study of spectral characterizations of graphs has developed significantly. Therefore, we believe that a second survey has become worthwhile. In this survey, we focus on new developments. Most of the mentioned results have been published, whereas some other results are new; obtained either by the authors themselves, or through personal communications.

We do not only consider the spectrum of the adjacency matrix, but also deal with the Laplacian matrix, the (so-called) signless Laplacian, and the generalized adjacency matrices. As in [13], we abbreviate 'determined by the spectrum' by DS.

An important development is the new method of Wang and Xu (see Section 5) for finding graphs that are DS with respect to the generalized adjacency matrix. Their approach often works for randomly generated graphs, and this strengthens our believe that the statement 'almost all graphs are not DS' (which is true for trees) is false.

Another result deals with cospectrality of generalized adjacency matrices, in particular an answer is given to the question (posed in [13]): 'when can regularity of a graph be deduced from the spectrum of a generalized adjacency matrix?' (see Section 4).

Several families of graphs are shown to be DS with respect to the adjacency matrix (see Section 2), the Laplacian matrix (see Section 3), or both (see Section 6.1). For the signless Laplacian we know of one new result (see Section 3). However, the remark made in [13] that, with respect to the signless Laplacian, graphs tend to be more often DS than with respect to the Laplacian, or (generalized) adjacency matrix, motivated Cvetković, Rowlinson, and Simić [12] to (re)start investigations of this rather unusual matrix.

For many other graphs, cospectral mates have been found. This includes some special bipartite graphs (see Section 2.3), and many distance-regular graphs (see Section 6.4). One such family of graphs cospectral with distance-regular graphs turned out to be a new infinite family of distance-regular graphs. Important methods for constructing cospectral graphs are Godsil-McKay switching [23] and the partial-linear-space technique, which have been explained in our previous survey [13]. We assume the reader to be familiar with the methods and results from that paper.

\footnotetext{
* Corresponding author.

E-mail addresses: Edwin.vanDam@uvt.nl (E.R.van Dam), Haemers@uvt.nl (W.H. Haemers).
} 


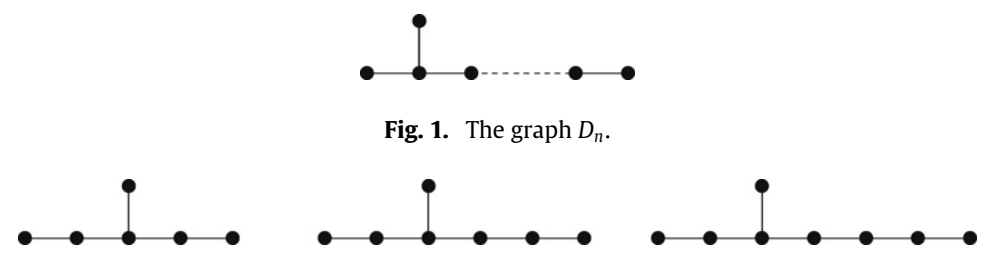

Fig. 2. The graphs $E_{6}, E_{7}, E_{8}$.

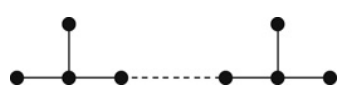

Fig. 3. The graph $\tilde{D}_{n}$.
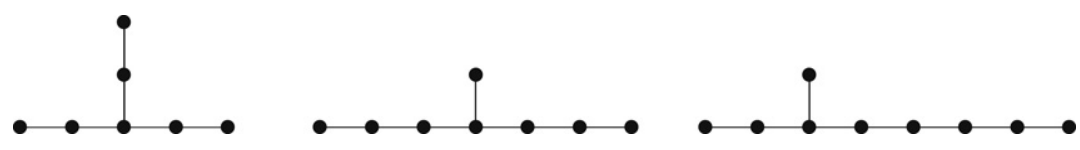

Fig. 4. The graphs $\tilde{E}_{6}, \tilde{E}_{7}, \tilde{E}_{8}$.

\section{The adjacency matrix}

\subsection{Graphs with small spectral radius}

The recent results on graphs that are determined by the adjacency spectrum are dominated by results for graphs with small spectral radius. Using Smith's [37] classification of graphs with spectral radius less than 2, it was determined by Shen et al. [36] that all connected such graphs - the paths $P_{n}$ of size $n$, the graphs $D_{n}$ of size $n$, and the graphs $E_{6}, E_{7}$, and $E_{8}$ (see Figs. 1 and 2) - are DS.

Shen et al. [36] also pointed out a mistake in [13] where we claimed that a disjoint union of paths is DS. This is only so if all paths have size at least two, i.e., if there are no isolated vertices (paths of size 1). Indeed, the disjoint union of $P_{2 n+1}$ and $P_{1}$ is cospectral with the disjoint union of $D_{n+2}$ and $P_{n}$, cf. [36]. This fact is easily shown by using the partitioned tensor product method of Godsil and McKay [24], who illustrate the case $n=2$ in their Example 2.5. Other examples spoiling the general claim are $P_{11}+P_{2}+P_{1}$ which is cospectral with $E_{6}+P_{5}+P_{3}$, and $P_{17}+P_{2}+P_{1}$ which is cospectral with $E_{7}+P_{8}+P_{5}$, and $P_{29}+P_{4}+P_{2}+P_{1}$ which is cospectral with $E_{8}+P_{14}+P_{9}+P_{5}$.

Shen et al. [36] finally showed that a disjoint union of graphs of type $D_{n}, n \geq 4$ is DS. Although the classification of DS graphs with spectral radius less than 2 may not be complete, we think that the most prominent questions here have been answered. One final remark that could be made is that if a graph is cospectral with a graph with spectral radius less than 2 , then it has the same number of components (since the number of edges is the same), and also the same number of components that are paths of size at least two. The latter follows from considering the sum of squares of degrees in the graph, which is determined by the spectrum in the absence of 4-cycles.

Smith [37] also determined all connected graphs with spectral radius 2: the cycles $C_{n}$ of size $n$, the graphs $\tilde{D}_{n}$ of size $n+1 \geq 5$, and the graphs $\tilde{E}_{6}, \tilde{E}_{7}, \tilde{E}_{8}$ (see Figs. 3 and 4). It is well-known that the cycles are DS. It is also known that the graphs $\overline{\tilde{D}}_{n}$ are cospectral with the disjoint union of a 4-cycle and a path $P_{n-3}$, cf. [11, p. 77]. The graph $\tilde{E}_{6}$ is cospectral with the disjoint union of a 6-cycle and an isolated vertex, while the remaining two graphs, $\tilde{E}_{7}$ and $\tilde{E}_{8}$ are DS (this follows among others from the results below). We may thus conclude the following.

Proposition 1. All connected graphs with spectral radius at most 2 are DS except for the graphs $\tilde{D}_{n}, n \geq 4$ and $\tilde{E}_{6}$.

Wang and $\mathrm{Xu}$ [41] determined the DS graphs among the so-called T-shape trees, that is, the trees with one vertex of maximal degree 3 . Such trees have spectral radius less than $\frac{3}{2} \sqrt{2} \approx 2.1312$. In their analysis, Wang and Xu among others use that in a graph without 4-cycles, the number of 2-matchings is a coefficient in the characteristic polynomial. Let $T(a, b, c)$ denote the T-shape tree, such that removal of the vertex of degree 3 leaves paths of sizes $a, b$, and $c$. Then Wang and Xu [41] showed the following.

Proposition 2. The T-shape tree $T(a, b, c)$ is $D S$ if and only if $\{a, b, c\} \neq\{d, d, 2 d-2\}$ for any $d$.

The exceptional graph $T(d, d, 2 d-2)$ is cospectral with the disjoint union of a path of size $d-1$ and the graph obtained by adding an edge between (one of the vertices of) a cycle of size $2 d+2$ and one of the end vertices of a path of size $d-2$ (a so-called lollipop graph, see below).

Ghareghani et al. [22] used the characterization of graphs with spectral radius between 2 and $\sqrt{2+\sqrt{5}} \approx 2.0582$ by Brouwer and Neumaier [4], to determine that all such connected graphs are DS. These comprise a collection of T-shape trees and a collection of trees with two vertices of maximal degree 3. 
Proposition 3. All connected graphs with spectral radius between 2 and $\sqrt{2+\sqrt{5}}$ are DS.

In [25] it is proved that so-called lollipop graphs with an odd cycle are DS. A lollipop graph is a graph obtained by adding an edge between (one of the vertices of) a cycle and one of the end vertices of a path. Such graphs have spectral radius at most $\sqrt{5}$. Also the lollipop graphs with an even cycle are DS (Tayfeh-Rezaie [private communication] did the lollipop graphs with a cycle of length at least 6 , and Boulet and Jouve [3] did the general case.). Finally we would like to mention that Woo and Neumaier [45] obtained some results on graphs with spectral radius at most $\frac{3}{2} \sqrt{2}$ (among them are for example all T-shape trees, and lollipop graphs with sufficiently large cycle length and fixed path length). These results could be useful in the further classification of DS graphs with small spectral radius. We do not consider this the most important challenge though.

It would, for example, be more interesting to determine which starlike trees (trees with one vertex of degree larger than 2) are determined by the spectrum. Partial results are obtained by Lepović and Gutman [31] who showed that no starlike trees are cospectral, and by Omidi and Tajbakhsh [35] who showed that if a tree is cospectral with, but not isomorphic to a given starlike tree, then its maximum degree is less than the maximum degree of the starlike tree, and any two vertices of degree at least five are adjacent (and hence there can be at most two).

\subsection{Bipartite graphs with few eigenvalues}

In [17], nonregular bipartite graphs with four distinct eigenvalues were studied. Among others all such connected (connectivity follows from the spectrum in this case) graphs on at most 60 vertices were determined. Thirteen of these graphs are DS, the smallest of which is the path on four vertices.

In [18], bipartite biregular graphs with five eigenvalues were studied. All such connected graphs on at most 33 vertices were determined. However, biregularity is not a property that is determined by the spectrum. Regularity is determined by the spectrum though, and thus among the above graphs we find five regular DS graphs (where graphs whose adjacency matrix has rank 6 are excluded, see below). Three of these are distance-regular: the Pappus graph, Tutte's 8-cage, and the incidence graph of the affine plane of order 4 minus a parallel class (see also [13, Table 4]). It would be interesting to find out whether the other unique biregular graphs are DS.

In the above, graphs of small rank are excluded. It also follows from the results in [18] that the regular graphs $C_{6} \otimes J_{t}$ and $C_{8} \otimes J_{t}$ are DS. The problem to determine (all) other (DS) graphs with small rank (among the bipartite graphs with five eigenvalues) also seems feasible.

In general, it should be possible to determine the DS graphs among the graphs whose adjacency matrix has very small rank. The disjoint union of a complete bipartite graph and some isolated vertices is for example not DS (in general). However, it was observed by Stevanović and the authors [private communication] that the complement of such a graph is always DS. If $A$ is the adjacency matrix of this complement, then $A+I$ has rank 3. Indeed, it also seems feasible to determine the DS graphs for which almost all eigenvalues are -1 .

\subsection{Cospectral bipartite graphs with different bipartitions}

At the Aveiro Workshop on Graph Spectra in 2006, Zwierzyński [38, Problem AWGS.2.B] asked if one can determine the size of a bipartition given the spectrum of a connected bipartite graph. In general, the answer to this question is negative, and an infinite family of examples where the bipartition sizes differ by one were given in [38, Problem AWGS.2.B]. Here we give an infinite family of cospectral connected bipartite graphs, for which the bipartition sizes differ by an arbitrary amount, and for which also the complements are cospectral.

Consider the connected bipartite graph $G$ with incidence matrix

$\left[\begin{array}{lllllll}J & J & O & O & O & J & J \\ J & O & J & O & J & O & J \\ J & O & O & J & J & J & O\end{array}\right] \begin{gathered}n_{1} \\ n_{2} \\ n_{3}\end{gathered}$

where $J$ and $O$ denote all-ones matrices and all-zeros matrices, respectively, of sizes indicated by the variables besides and below the matrix. The set of vertices corresponding to the first $4 \mathrm{~m}$ columns induce a subgraph that satisfies the conditions for Godsil-McKay switching, and switching gives a cospectral bipartite graph $H$, which has incidence matrix

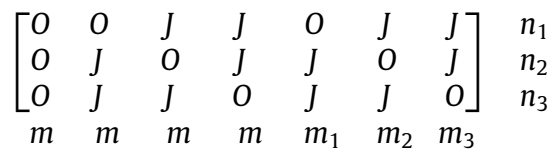

but which is not connected. Still, let $k_{i}, i=1,2,3$ be integers greater than $m$, let $G_{1}$ be the graph with $m_{i}=0, i=1,2,3$ and $n_{i}=k_{i}, i=1,2,3$, and let $H_{1}$ be its switched graph. Also, let $G_{2}$ be the graph with $m_{i}=k_{i}-m, i=1,2,3$ and $n_{i}=m, i=1,2,3$, and let $H_{2}$ be its switched graph. Then $H_{1}$ and $H_{2}$ are isomorphic graphs, and hence $G_{1}$ and $G_{2}$ are cospectral graphs whose complements are also cospectral. Moreover, $G_{1}$ has parts of sizes $4 m$ and $k_{1}+k_{2}+k_{3}$, while $G_{2}$ has parts of sizes $m+k_{1}+k_{2}+k_{3}$ and $3 m$. This example is further illustrated in Figs. 5 and 6. 

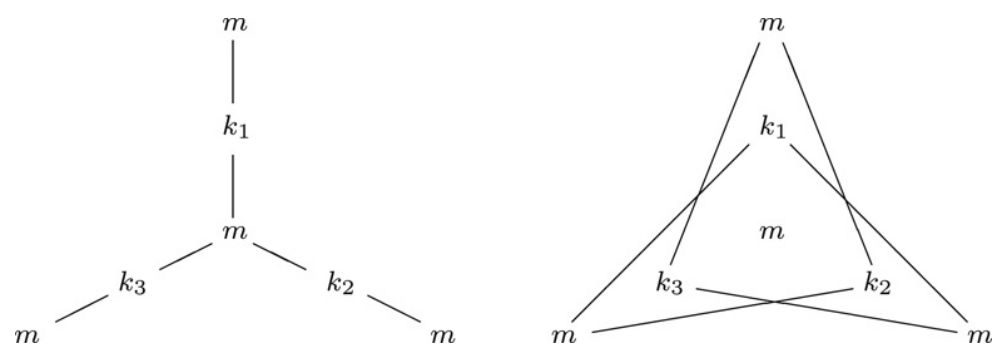

Fig. 5. Quotients of switching equivalent graphs $G_{1}$ and $H_{1}$.
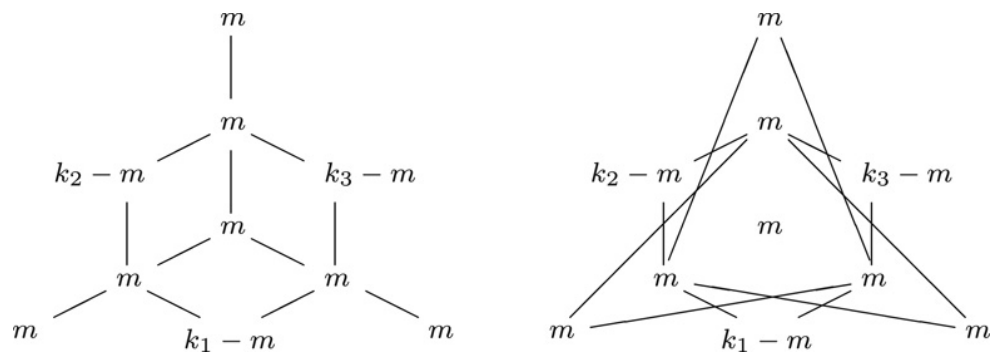

Fig. 6. Quotients of switching equivalent graphs $G_{2}$ and $H_{2}$.

\section{The Laplacian matrix}

It is known that the spectrum of the Laplacian matrix of a graph determines the number of vertices, the number of edges, the number of components, and the number of spanning trees, cf. [13, Lemma 4]. Also the sum of squares of degrees of a graph follows from the Laplacian spectrum, as can be seen from the proof of Proposition 2 of [13]. For bipartite graphs it can even be shown that the sum of cubes of degrees is determined by the Laplacian spectrum. This follows from the following.

Lemma 1. Let $G$ be a bipartite graph with $n$ vertices and $m$ edges, and with Laplacian eigenvalues $\theta_{i}, i=1, \ldots, n$ and vertex degrees $d_{i}, i=1, \ldots, n$. Then $\sum_{i}\left(\begin{array}{c}d_{i} \\ 3\end{array}\right)=\frac{1}{6} \sum_{i}\left(\theta_{i}-2\right)^{3}+\frac{1}{6}(m-n)(-2)^{3}$.

Proof. If $N$ is the $(0, \pm 1)$ vertex-edge incidence matrix of $G$, with ones in one part of the bipartition, and minus ones in the other part, then $N N^{\top}$ is the Laplacian matrix of $G$, while $N^{\top} N-2 I$ is the adjacency matrix $A$ of its line graph. By counting the number of triangles in the line graph, which equals $\frac{1}{6}$ trace $\left(A^{3}\right)$, the result follows.

The problem in using this lemma is that bipartiteness is a property that is not determined by the Laplacian spectrum, at least not in general. However, trees are bipartite graphs, and it is determined by the Laplacian spectrum whether a graph is a tree (and hence bipartite). Lemma 1 is essentially what finishes the proof of Omidi and Tajbakhsh [35] that starlike trees are determined by the Laplacian spectrum, a proof that builds on the result by Lepović [30] that no two starlike trees have the same Laplacian spectrum.

Shen et al. [36] used the fact that if $\theta_{1}$ is the largest Laplacian eigenvalue of a non-empty graph, then the degree $d$ of a vertex is at most $\theta_{1}-1$ (this follows from interlacing, and the fact that $K_{1, d}$ is a subgraph). This, and the above remarks determine the degree sequence for graphs cospectral with the tree $\tilde{D}_{n}$ (see Fig. 3 ). They then also used counting arguments in the line graph (the sum of squares of degrees, in the absence of 4-cycles) to show that $\tilde{D}_{n}$ is determined by the Laplacian spectrum.

The same arguments are used in [25] to determine the degree sequence of a graph cospectral with a lollipop graph (as defined in Section 2.1). By counting the number of spanning trees, it is then shown that every lollipop graph is determined by the Laplacian spectrum.

The relation with the line graph can also be explored when the so-called signless Laplacian matrix (cf. [13]) is considered. Omidi [private communication] used this to show that all the T-shape trees except $K_{1,3}$ are determined by the signless Laplacian spectrum.

A fan graph is a cone over a path, that is, the graph obtained from a path by adding a vertex that is adjacent to all vertices of the path. Similarly a wheel graph is a cone over a cycle. Liu et al. proved that fan graphs [32] and odd wheel graphs [private communication] are determined by the Laplacian spectrum. In their proof, they use that the largest Laplacian eigenvalue is at most $d_{1}+d_{2}$, the sum of the largest two vertex degrees (which follows by considering the correspondence with the line graph, which has vertex degrees at most $d_{1}+d_{2}-2$ ). By using this, and also the second largest Laplacian eigenvalue, they manage to limit the number of possible degree sequences for graphs that are cospectral with a fan graph or an odd wheel graph, which finally leads to the proof that these graphs are determined by the Laplacian spectrum. 
Hammer and Kelmans [28] proved that so-called threshold graphs are determined by the Laplacian spectrum. Threshold graphs are defined in terms of stable sets, but they are the same as the 1-decomposable graphs. A graph is called 1decomposable if it is obtained from a single vertex by alternatingly adding isolated vertices and taking complements. The key idea in their proof can be generalized as follows.

Lemma 2. Let $G$ be a graph on $n$ vertices with largest Laplacian eigenvalue $n$. If $G$ is determined by the Laplacian spectrum, then the graph $H$ obtained from $G$ by adding $m$ isolated vertices is also determined by the Laplacian spectrum.

Proof. Consider a graph with the same spectrum as $H$. Then it has $n+m$ vertices, the same number of components as $H$, and largest Laplacian eigenvalue $n$. This implies that it has a component of size at least $n$. Since there are at least $m$ other components (of sizes at least one), and there is a total of $n+m$ vertices, it follows that it has one component of size $n$, and $m$ isolated vertices. The component of size $n$ then has the same Laplacian spectrum as $G$, hence it must be isomorphic to $G$. Thus $H$ is determined by its Laplacian spectrum.

By using that a graph is determined by the Laplacian spectrum if and only if its complement is, and that a graph on $n$ vertices has Laplacian eigenvalue $n$ if and only if its complement is disconnected, we derive the following.

Proposition 4. Let $G$ be a disconnected graph that is determined by the Laplacian spectrum. Then the cone over $G$, the graph $H$ that is obtained from $G$ by adding one vertex that is adjacent to all vertices of $G$, is also determined by its Laplacian spectrum.

Proof. This follows from Lemma 2 and the above remarks, because the complement of $G$ has largest eigenvalue equal to the number of vertices and the complement of $H$ is the complement of $G$ with an isolated vertex.

Since the disjoint union of paths, and also the disjoint union of cycles is determined by the Laplacian spectrum (cf. [13]), it follows that the cones over these graphs, the so-called multi-fan graphs (cf. [32]) and multi-wheel graphs are determined by the Laplacian spectrum.

\section{Cospectral generalized adjacency matrices}

One of the open problems mentioned in [13] concerns the generalized adjacency matrix. If $A_{G}$ is the adjacency matrix of a graph $G$, any matrix of the form $M=\alpha A_{G}+\beta J+\gamma I$ with $\alpha, \beta, \gamma \in \mathbb{R}$ and $\alpha \neq 0$ is called a generalized adjacency matrix of $G$ (as usual, $J$ denotes the all-ones matrix and $I$ the identity matrix). Note that for graphs $G$ and $G^{\prime}, \alpha A_{G}+\beta J+\gamma I$ and $\alpha A_{G^{\prime}}+\beta J+\gamma I$ (with $\alpha \neq 0$ ) are cospectral if and only if $A_{G}-y J$ and $A_{G^{\prime}}-y J$ with $y=-\beta / \alpha$ are cospectral. So without loss of generality we may restrict to generalized adjacency matrices of the form $A_{G}-y J$. Note also that if $\bar{G}$ is the complement of $G$, then $y J-A_{\bar{G}}=A_{G}-(1-y) J+I$. Thus if $A_{G}-y J$ and $A_{G^{\prime}}-y J$ are cospectral, then so are $A_{\bar{G}}-(1-y) J$ and $A_{\bar{G}^{\prime}}-(1-y) J$.

It is well known that with respect to the usual adjacency matrix $A_{G}$, a regular graph cannot be cospectral with a nonregular graph, cf. [11, p. 94]. For generalized adjacency matrices the following result was proved in [13].

Proposition 5. Suppose $G$ is a regular graph, and $G^{\prime}$ is a non-regular graph. Then the generalized adjacency matrices $A_{G}-y J$ and $A_{G^{\prime}}-y J$ cannot be cospectral, except possibly when $0<y<1$.

For $y=\frac{1}{2}$, every regular graph $G$ with at least three vertices is cospectral with a non-regular graph. Indeed, multiplication of some rows and the corresponding columns of $A_{G}-\frac{1}{2} J$ with -1 gives a cospectral matrix, which corresponds to another graph $G^{\prime}$ (the operation is called Seidel switching). And if $G$ has at least three vertices, one can always choose rows (and columns) such that $G^{\prime}$ is non-regular graph. For example the triangle (which is regular) and the graph on three vertices with one edge (which is non-regular) are cospectral for $y=\frac{1}{2}$. When [13] was written, it was an open problem whether the above statement is true or false for $0<y<1, y \neq \frac{1}{2}$. Now we know the answer for all values of $y$.

Theorem 1. There exists a pair of graphs $G$ and $G^{\prime}$, one regular and one not, for which the matrices $A_{G}-y J$ and $A_{G^{\prime}}-y J$ are cospectral if and only if $y$ is a rational number satisfying $0<y<1$.

The construction of such pairs of graphs for the given values of $y$ is given in [8]. In [14] all such pairs on at most eleven vertices are generated. The smallest such pair of graphs with $y \neq \frac{1}{2}$ has $y=\frac{1}{3}$ and is presented in Fig. 7. The fact that such a pair cannot exist for irrational $y$ follows from the following theorem.

Theorem 2. For two graphs $G$ and $G^{\prime}$, the following are equivalent:

i. $A_{G}-y J$ and $A_{G^{\prime}}-y J$ are cospectral for all values of $y$,

ii. $A_{G}-y \mathrm{~J}$ and $A_{G^{\prime}}-y \mathrm{~J}$ are cospectral for two distinct values $y$,

iii. $A_{G}-y J$ and $A_{G^{\prime}}-y J$ are cospectral for an irrational value of $y$.

Indeed, by this theorem cospectrality for an irrational $y$ implies cospectrality for the adjacency matrix, and hence $G$ is regular if and only if $G^{\prime}$ is. Equivalence of $i$ and $i i$ is due to Johnson and Newman [29]. The following short proof for the above theorem appeared in [14]. 


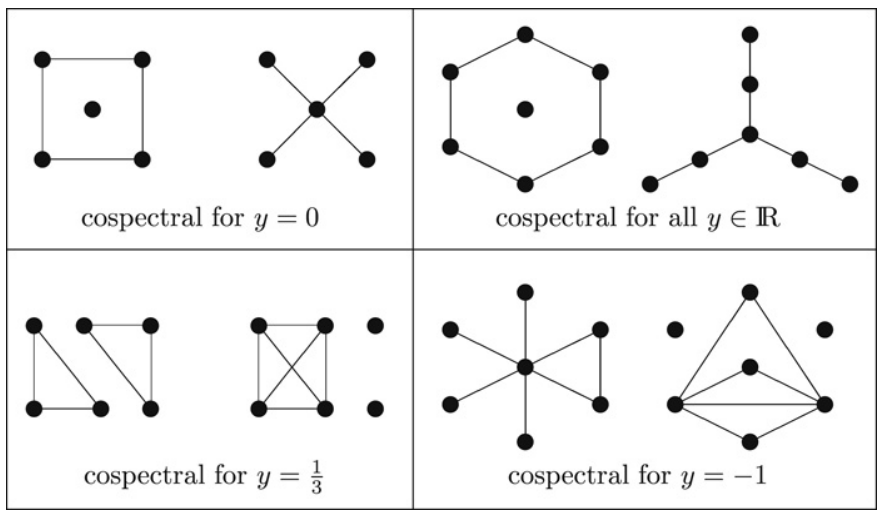

Fig. 7. Examples of pairs of graphs with cospectral matrices $A_{G}-y J$.

Proof. For a graph $G$ we define the generalized characteristic polynomial: $p_{G}(x, y)=\operatorname{det}\left(x I+y J-A_{G}\right)$. Thus $p_{G}(x, y)$ can be interpreted as the characteristic polynomial of $A_{G}-y J$, and $p_{G}(x, 0)$ is the characteristic polynomial of $A_{G}$. Moreover, $p_{G}(x, y)$ has integral coefficients. It follows that the degree in $y$ of $P_{G}(x, y)$ is 1 . Indeed, for an arbitrary square matrix $M$ it is known that $\operatorname{det}(M+y J)=\operatorname{det} M+y \Sigma \operatorname{adj} M$, where $\Sigma \operatorname{adj} M$ denotes the sum of the entries of the adjugate (adjoint) of $M$. It is also easily derived from the fact that by Gaussian elimination in $x I+A_{G}-y J$ one can eliminate all $y$ 's, except for those in the first row. So we may write

$$
p_{G}(x, y)=\sum_{i=0}^{n}\left(a_{i}+b_{i} y\right) x^{i} .
$$

It is clear that $p_{G}(x, y) \equiv p_{G^{\prime}}(x, y)$ if and only if $A_{G}-y J$ and $A_{G^{\prime}}-y J$ are cospectral for all $y \in \mathbb{R}$, and that $A_{G}-y J$ and $A_{G^{\prime}}-y J$ are cospectral for some, but not all, values of $y$ if and only if $p_{G}(x, y)=p_{G^{\prime}}(x, y)$ for all $x \in \mathbb{R}$, whilst $p_{G}(x, y) \not \equiv p_{G^{\prime}}(x, y)$. If this is the case, then $a_{i}+y b_{i}=a_{i}^{\prime}+y b_{i}^{\prime}$ with $b_{i} \neq b_{i}^{\prime}$ for some $i$. This implies $y=-\left(a_{i}-a_{i}^{\prime}\right) /\left(b_{i}-b_{i}^{\prime}\right)$. Thus we proved that $y$ is rational, and that there is only one possible value of $y$.

If $A_{G}-y J$ and $A_{G}-y J$ are cospectral for all $y \in \mathbb{R}$, we say that $G$ and $G^{\prime}$ are cospectral for the generalized spectrum. From the theorem above it follows that this happens if and only if $G$ and $G^{\prime}$ are cospectral with cospectral complements. This implies that cospectral graphs that are constructed via Godsil-McKay switching are cospectral for the generalized spectrum. The upper right pair of graphs in Fig. 7 is of this type. For the other ones, $A_{G}-y J$ and $A_{G^{\prime}}-y J$ are cospectral only for the given value of $y$. In [14], it is proved that such a pair exists for every rational $y$. All such pairs on at most nine vertices are also enumerated there.

\section{Graphs determined by their generalized spectrum}

Though it seems that many graphs are DS, it is difficult to prove this property for a given graph. In [13] two methods are used. One is complete enumeration of all graphs on $n$ vertices $(n \leq 11)$. The other approach uses structural properties that characterize the graph and that can be deduced from the spectrum. Recently Wang and Xu $[40,42,43]$ presented a new method, which we will briefly explain in this section.

The method of Wang and Xu is based on the following idea. If $G$ and $G^{\prime}$ are cospectral, then the matrices $A_{G}$ and $A_{G^{\prime}}$ are similar, so there exist an orthogonal matrix $Q$ such that $Q^{\top} A_{G} Q=A_{G^{\prime}}$. If $Q$ is a permutation matrix, $G$ and $G^{\prime}$ are isomorphic. So one could try to show that $G$ is DS by generating all orthogonal matrices $Q$ such that $Q^{\top} A_{G} Q$ is a $(0,1)$-matrix. If all these $Q$ are permutation matrices, $G$ is clearly DS. At first glance this approach looks hopeless. Wang and Xu made it feasible by making two major assumptions:

- The graph $G$ is required to be determined by the spectrum together with the spectrum of the complement. By Theorem 2 this is the case if and only if $G$ is determined by the spectra of its generalized adjacency matrices, and we say that $G$ is determined by the generalized spectrum and abbreviate it by $G$ being DGS. Thus a graph $G$ is DGS whenever every graph that is cospectral with $G$ for the generalized spectrum, is isomorphic to G. So DGS is a weaker property than DS; for example $K_{1,4}$ is DGS, but not DS (see Fig. 7).

- Only a special class of graphs called $g_{n}$ is considered. This is the set of graphs on $n$ vertices whose adjacency matrix has no eigenvector orthogonal to the all-ones vector $\mathbf{1}$. We will see that graphs in this class are highly irregular, and in particular they have trivial automorphism group. This makes the method especially suitable for graphs for which the structural approach is not successful.

An important tool in the approach of Wang and Xu is the $n \times n$ walk matrix $W_{G}$ of a graph $G$ on $n$ vertices, defined by

$$
W_{G}=\left[\mathbf{1}, A_{G} \mathbf{1}, A_{G}^{2} \mathbf{1}, \ldots, A_{G}^{n-1} \mathbf{1}\right] .
$$


Suppose that $P_{G}(x)=\sum_{i=0}^{n} c_{i} x^{i}$ is the characteristic polynomial of $A_{G}$, and let $C_{G}$ be its companion matrix, that is,

$$
C_{G}=\left[\begin{array}{cc}
\mathbf{0}^{\top} & -c_{0} \\
I_{n-1} & -\mathbf{c}
\end{array}\right], \quad \text { with } \mathbf{c}=\left[\begin{array}{llll}
c_{1} & c_{2} & \ldots & c_{n-1}
\end{array}\right]^{\top} .
$$

Then $A_{G}^{n}=-\sum_{i=0}^{n-1} c_{i} A_{G}^{i}$ (by the Cayley-Hamilton theorem), and therefore

$$
A_{G} W_{G}=\left[A_{G} \mathbf{1}, A_{G}^{2} \mathbf{1}, \ldots, A_{G}^{n} \mathbf{1}\right]=W_{G} C_{G} .
$$

The matrix $W_{G}$ is called walk matrix because $\left(W_{G}\right)_{i, j}$ gives the number of walks of length $j-1$ that start in vertex $i$. In particular $\mathbf{1}^{\top} A_{G}^{j-1} \mathbf{1}$, which is the $j$ th entry of $\mathbf{1}^{\top} W_{G}$, gives the total number of walks in $G$ of length $j-1$.

Lemma 3. Suppose $G$ and $G^{\prime}$ are cospectral graphs with cospectral complements. Then

$$
W_{G}^{\top} W_{G}=W_{G^{\prime}}^{\top} W_{G^{\prime}} \text {. }
$$

Proof. We use the fact (see for example [11]) that for every positive integer $j$, the total number of walks of length $j$ can be expressed in terms of the characteristic polynomial of the graph and the characteristic polynomial of its complement. Therefore these numbers are the same for $G$ and $G^{\prime}$, hence $\mathbf{1}^{\top} A_{G}^{j} \mathbf{1}=\mathbf{1}^{\top} A_{G^{\prime}}^{j} \mathbf{1}$ for every positive integer $j$. It follows that

$$
\left(W_{G}^{\top} W_{G}\right)_{i, j}=\mathbf{1}^{\top} A_{G}^{i+j-2} \mathbf{1}=\mathbf{1}^{\top} A_{G^{\prime}}^{i+j-2} \mathbf{1}=\left(W_{G^{\prime}}^{\top} W_{G^{\prime}}\right)_{i, j} .
$$

If $G$ is regular of degree $k$, then $W_{G}$ has rank 1 and depends only on $k$. On the other hand, for many graphs $W_{G}$ turns out to be non-singular. This is precisely the case if $G \in g_{n}, \mathrm{cf}$. [27].

Theorem 3. Suppose $G$ and $G^{\prime}$ are cospectral graphs with cospectral complements and let $G \in g_{n}$. Then $G^{\prime} \in g_{n}$ and $Q=\left(W_{G^{\prime}} W_{G}^{-1}\right)^{\top}$ is the unique orthogonal matrix such that $A_{G^{\prime}}=Q^{\top} A_{G} Q$ and $Q \mathbf{1}=\mathbf{1}$.

Proof. The orthogonality of $Q$ follows straightforwardly from Lemma 3. Therefore $Q$ is invertible, and so is $W_{G^{\prime}}$, hence $G^{\prime} \in g_{n}$. Since $G$ and $G^{\prime}$ are cospectral, the companion matrices $C_{G}$ and $C_{G^{\prime}}$ are equal, hence (1) gives $A_{G^{\prime}}=Q^{\top} A_{G} Q$. To see uniqueness, observe that $A_{G^{\prime}}=Q^{\top} A_{G} Q$ implies $Q^{\top} A_{G}^{i}=A_{G^{\prime}}^{i} Q^{\top}(i=1,2, \ldots)$, and by use of $Q^{\top} \mathbf{1}=Q \mathbf{1}=\mathbf{1}$ this gives $Q^{\top} W_{G}=W_{G^{\prime}}$, hence $Q^{\top}=W_{G^{\prime}} W_{G}^{-1}$.

In the special case that $G=G^{\prime}$, we have $A_{G}=P A_{G} P^{\top}$ for some unique permutation matrix $P$. So $P=I$, hence $G$ has no nontrivial automorphisms. Therefore all graphs in $g_{n}$ have trivial automorphism group.

The important conclusion from the above theorem is that $Q$ is a rational matrix (because the walk matrix is integral). For a rational matrix $Q$ we define the level to be smallest positive integer $\ell$ such that $\ell Q$ is an integral matrix. For a given graph $G \in g_{n}$, the class $Q_{G}$ is defined to be the class of orthogonal rational matrices $Q$ that satisfy $Q \mathbf{1}=\mathbf{1}$, and $Q^{\top} A_{G} Q$ is a $(0,1)$-matrix. Note that $Q^{\top} A_{G} Q$ is symmetric with zero diagonal (because trace $A_{G}=\operatorname{trace} Q^{\top} A_{G} Q$ ). It is clear that an integral orthogonal matrix is a permutation matrix, so if every $Q \in Q_{G}$ has level 1 , then $G$ is DGS. From the theorem above, it follows that $Q^{\top}=W_{G^{\prime}} W_{G}^{-1}$ for every $Q \in \mathcal{Q}_{G}\left(G^{\prime}\right.$ is the graph with adjacency matrix $\left.Q^{\top} A_{G} Q\right)$, therefore $\ell \mid \operatorname{det} W_{G}$. But we can be more precise by considering the Smith normal form of $W_{G}$. Recall that the Smith normal form of $W_{G}$ is the integral diagonal matrix $D=\operatorname{diag}\left(d_{1}, \ldots, d_{n}\right)$, such that $d_{1}\left|d_{2}\right| \ldots \mid d_{n}$, and $D=U W_{G} V$ for unimodular matrices $U$ and $V$. It follows that $W_{G}^{-1}=V D^{-1} U$, hence $d_{n} W_{G}^{-1}$ is integral and therefore $\ell \mid d_{n}$. The next lemma is used to reduce the possible values of $\ell$ even further:

Lemma 4. Suppose $Q \in Q_{G}$ with level $\ell$. If $p$ is a prime factor of $\ell$, then the following congruences have an integral solution $\mathbf{z} \not \mathbf{0}(\bmod p)$.

$$
W_{G}^{\top} \mathbf{z} \equiv \mathbf{0}, \quad \mathbf{z}^{\top} \mathbf{z} \equiv 0(\bmod p) .
$$

Proof. Let $\mathbf{z} \neq \equiv \mathbf{0}(\bmod p)$ be a column of $\ell Q$. Such a column exits, since otherwise $\ell Q / p$ would be an integral matrix which contradicts the minimality of $\ell$. Observe that $W_{G}^{\top} \mathbf{z}$ is a column of $\ell W_{G}^{\top} Q$. By Theorem $3, Q^{\top} W_{G}=W_{G^{\prime}}$, so $W_{G}^{\top} Q$ is an integral matrix, and therefore $W_{G}^{\top} \mathbf{z} \equiv \mathbf{0}(\bmod p)$. Moreover, $Q^{\top} Q=I$ implies that $\mathbf{z}^{\top} \mathbf{z}=\ell^{2} \equiv 0(\bmod p)$.

It turns out that this lemma cannot exclude 2 from being a prime factor of $\ell$, but odd prime factors of $d_{n}$ very often can be evaluated. For that reason we define the subclass $\mathscr{H}_{n}$ of $g_{n}$, consisting of those graphs in $g_{n}$ for which $d_{n} \equiv 2(\bmod 4)$, and for which all odd prime factors of $d_{n}$ are excluded by the conditions of the above lemma. We have that $\ell \leq 2$ for all graphs in $\mathscr{H}_{n}$. This makes it feasible to determine all matrices in $\mathcal{Q}_{G}$ for graphs $G \in \mathscr{H}_{n}$. Note that finding the set $\mathcal{Q}_{G}$ leads to all graphs cospectral with $G$ with respect to the generalized spectrum. In [42], Wang and Xu present an explicit algorithm based on these ideas. They find (among others) an example of a randomly generated graph on 24 vertices which is DGS. In [40], the above results are used to obtain the following sufficient conditions for a graph to be DGS. 
Theorem 4. Suppose $G \in g_{n}$, and let $D=\operatorname{diag}\left(d_{1}, \ldots, d_{n}\right)$ be the Smith normal form of the walk matrix $W_{G}$. Let $U$ and $V$ be unimodular matrices such that $D=U W_{G} V$, and let $\mathbf{u}^{\top}$ be the last row of $U$. If $d_{n} \equiv 2(\bmod 4), \operatorname{gcd}\left(\mathbf{u}^{\top} \mathbf{u}, d_{n} / 2\right)=1$, and $W_{G} \mathbf{z} \equiv \mathbf{0}(\bmod 2)$ for every $(0,1)$-vector $\mathbf{z}$ with weight 4 (i.e. $\mathbf{z}$ contains exactly four 1's), then $G$ is DGS.

In [43], Wang and Xu used their approach to find conditions for which a DGS graph remains DGS if an isolated vertex is added. They obtain the following theorem.

Theorem 5. Let $G \in g_{n}$. If $\operatorname{gcd}\left(\operatorname{det} A_{G}\right.$, det $\left.W_{G}\right)=1$, then the graph obtained from $G$ by adding an isolated vertex is DGS if and only if $G$ is.

There is some evidence (no proof yet; see [42]) that, with high probability, a random graph $G$ on $n$ vertices is in $\mathscr{H}_{n}$. If $G \in \mathscr{H}_{n}$ has a non-isomorphic cospectral mate, then the corresponding orthogonal matrix $Q$ has level $\ell=2$. But the orthogonal matrices with $\ell=2$ and row sum 1 are known (see [42]), and if $Q^{\top} A_{G} Q$ is a $(0,1)$-matrix for such an orthogonal matrx $Q$, then $G$ must have a special structure. Therefore it seems safe to conjecture that the statement 'almost all graphs are not DGS' (which is true for trees, and strongly regular graphs; see next section) is false for graphs in general.

In [26] (see also [13]) all DS graphs and DGS graphs on at most eleven vertices are enumerated by computer. It turns out that the majority of pairs of graphs on at most eleven vertices that are cospectral for the generalized spectrum come from Godsil-McKay switching. However, in almost all cases, adjacency matrices related by Godsil-McKay switching are similar by an orthogonal matrix $Q$ for which $Q \mathbf{1}=\mathbf{1}$ and $2 Q$ is integral. Therefore, the computer results from [26] support the believe that most graphs on $n$ vertices are in $\mathscr{H}_{n}$.

\section{Distance-regular graphs}

In the study of distance-regular graphs, characterization is an important topic. Much effort goes to characterization in terms of the parameters (intersection array), but characterization by the spectrum is stronger: if a distance-regular graph is determined by its spectrum, then it is determined by its parameters, but the converse is not true in general. The converse is true, however, if the diameter equals two, meaning that the graph is strongly regular.

We recall that for a regular graph DS with respect to the adjacency matrix, the Laplacian matrix, the signless Laplacian, and the set of generalized adjacency matrices (DGS) are equivalent.

\subsection{Strongly regular graphs determined by their spectrum}

A connected strongly regular is the same as a distance-regular graph with diameter two. A disconnected strongly regular graph is a disjoint union of two or more complete graphs of the same size. The complement of a strongly regular graph is again strongly regular. A connected regular graph is strongly regular if and only if it has exactly three distinct eigenvalues. One of the eigenvalues is the degree. The other eigenvalues of a strongly regular graph are also integral, or are equal to $(-1 \pm \sqrt{n}) / 2$, where $n$ is the number of vertices. See [7] for these and more results on strongly regular graphs. In [13] we surveyed the strongly regular graphs known to be determined by their parameters, and therefore by their spectrum. Up to taking complements, there are three infinite families: the disjoint unions of two or more complete graphs $K_{m}(m \geq 2)$, and the line graphs of $K_{n}(n \geq 4, n \neq 8)$ and $K_{k, k}(k \geq 2, k \neq 4)$. In addition there is a small list of exceptional strongly regular graph that are DS. In the meantime Coolsaet and Degraer $[10,19,20]$ showed for four more strongly regular graphs that they are DS. Table 1 is the updated list of sporadic strongly regular graphs that are DS (up to taking complements). The graphs marked with a $*$ are new in this list.

\subsection{Graphs cospectral with strongly regular graphs}

Most known strongly regular graphs have many cospectral mates. See $[6,21,33]$ for constructions of large sets of cospectral strongly regular graphs. In fact, almost all strongly regular graphs are not DS. More precisely:

Proposition 6. Let $a_{n}$ be the number of non-isomorphic strongly regular graphs on at most $n$ vertices, and let $b_{n}$ be the number of strongly regular graphs on at most $n$ vertices that are DS. Then $b_{n} / a_{n} \rightarrow 0$ if $n \rightarrow \infty$.

Proof. The spectrum of a strongly regular graph is determined by three integral parameters: the number of vertices $n$, the degree $k$ and the number $\lambda$ of triangles through an edge. Obviously $0 \leq \lambda<k<n$, therefore $n^{3}$ is an upper bound for the number of different spectra for a strongly regular graph on at most $n$ vertices, so $b_{n} \leq n^{3}$. On the other hand, the number $a_{n}$ grows exponentially in $n$ (see [6]).

We believe that, except for the three mentioned infinite families, only finitely many strongly regular graphs are DS. The following observation supports this statement.

Proposition 7. For a fixed $r \geq 2$, there exist only finitely many connected strongly regular graphs with second largest eigenvalue $r$ which are DS. 
Table 1

The known sporadic strongly regular DS graphs (up to complements)

\begin{tabular}{|c|c|c|c|c|}
\hline$n$ & & Spectrum & & Name \\
\hline 5 & {$[2]^{1}$} & {$[(-1+\sqrt{5}) / 2]^{2}$} & {$[(-1-\sqrt{5}) / 2]^{2}$} & Pentagon \\
\hline 13 & {$[6]^{1}$} & {$[(-1+\sqrt{13}) / 2]^{6}$} & {$[(-1-\sqrt{13}) / 2]^{6}$} & Paley \\
\hline 16 & {$[5]^{1}$} & {$[1]^{10}$} & {$[-3]^{5}$} & Clebsch \\
\hline 17 & {$[8]^{1}$} & {$[(-1+\sqrt{17}) / 2]^{8}$} & {$[(-1-\sqrt{17}) / 2]^{8}$} & Paley \\
\hline 27 & {$[10]^{1}$} & {$[1]^{20}$} & {$[-5]^{6}$} & Schläfli \\
\hline 50 & {$[7]^{1}$} & {$[2]^{28}$} & {$[-3]^{21}$} & Hoffman-Singleton \\
\hline 56 & {$[10]^{1}$} & {$[2]^{35}$} & {$[-4]^{20}$} & Gewirtz \\
\hline 77 & {$[16]^{1}$} & {$[2]^{55}$} & {$[-6]^{21}$} & Local Higman-Sims \\
\hline 81 & {$[20]^{1}$} & {$[2]^{60}$} & {$[-7]^{20}$} & Local GQ $(3,9)$ \\
\hline 100 & {$[22]^{1}$} & {$[2]^{77}$} & {$[-8]^{22}$} & Higman-Sims \\
\hline *105 & {$[32]^{1}$} & {$[2]^{84}$} & {$[-10]^{20}$} & Goethals-Seidel \\
\hline 112 & {$[30]^{1}$} & {$[2]^{90}$} & {$[-10]^{21}$} & $G Q(3,9)$ \\
\hline *120 & {$[42]^{1}$} & {$[2]^{99}$} & {$[-12]^{20}$} & Goethals-Seidel \\
\hline *126 & {$[50]^{1}$} & {$[2]^{105}$} & {$[-13]^{20}$} & Goethals \\
\hline 162 & {$[56]^{1}$} & {$[2]^{140}$} & {$[-16]^{21}$} & Local McLaughlin \\
\hline *176 & {$[70]^{1}$} & {$[2]^{154}$} & {$[-18]^{21}$} & Goethals-Seidel \\
\hline 275 & {$[112]^{1}$} & {$[2]^{252}$} & {$[-28]^{22}$} & McLaughlin \\
\hline
\end{tabular}

Proof (Sketch). The result is trivial if $r$ is not an integer, and otherwise it is a direct consequence of some famous theorems. Neumaier [34] showed that all, but finitely many, connected strongly regular graphs with integral eigenvalue $r$ can be constructed from Steiner 2-designs with block size $r+1$, or from a set of $r-1$ mutually orthogonal Latin squares. For 2-designs with block size at least 3 there is a famous theorem of Wilson [44] stating that for a large enough size, several non-isomorphic 2-designs with the required parameters exist. Existence of sets of $r-1(r>2)$ mutually orthogonal Latin squares, provided the size is large enough was proved by Chowla, Erdös, and Straus [9]. Actually we need that there exist at least two non-isotopic sets of given size, which is not mentioned explicitly in the paper, but which is clear from their approach. Thus we can conclude that in the two infinite families, every strongly regular graph has a nonisomorphic cospectral mate, provided the number of vertices is big enough.

Note that for all known strongly regular graphs that are DS, the graph or its complement has second largest eigenvalue at most 2 .

\subsection{Cospectral graphs from strongly regular graphs}

The following observation (by Godsil [private communication]) provides a method to construct pairs of cospectral graphs.

Proposition 8. Suppose $G$ and $G^{\prime}$ are cospectral strongly regular graphs. Let $H$ and $H^{\prime}$ be induced subgraphs of $G$ and $G^{\prime}$, respectively. Suppose that $H$ and $H^{\prime}$ are cospectral with cospectral complements (so $H$ and $H^{\prime}$ are cospectral with respect to all generalized adjacency matrices). Then $G \backslash H$ (the subgraph of $G$ induced by the vertices not in $H$ ) and $G^{\prime} \backslash H^{\prime}$ are cospectral with respect to all generalized adjacency matrices.

Proof. Suppose $G$ and $G^{\prime}$ have $n$ vertices and eigenvalues $k$ (the degree), $r$, and $s(r>s)$. Write

$$
A_{G}=\left[\begin{array}{cc}
A_{H} & N^{\top} \\
N & A_{G \backslash H}
\end{array}\right], \quad \text { and } \quad \tilde{A}_{G}=A_{G}+\frac{r-k}{n} J=\left[\begin{array}{cc}
A_{H}+\frac{r-k}{n} J & N^{\top}+\frac{r-k}{n} J \\
N+\frac{r-k}{n} J & A_{G \backslash H}+\frac{r-k}{n} J
\end{array}\right] .
$$

Then $\widetilde{A}_{G}$ has only two distinct eigenvalues $r$ and $s$. This implies (see for example [5], Lemma 2.1) that the spectrum of $A_{G \backslash H}+\frac{r-k}{n} J$ only depends on the spectra of $\widetilde{A}_{G}$ and $A_{H}+\frac{r-k}{n} J$. In a similar way, the spectrum of $A_{G^{\prime}} \backslash H^{\prime}+\frac{r-k}{n} J$ only depends on the spectra of $A_{G^{\prime}}+\frac{r-k}{n} J$ and $A_{H^{\prime}}+\frac{r-k}{n} J$. But the pair $\left\{\tilde{A}_{G}, A_{G^{\prime}}+\frac{r-k}{n} J\right\}$ is cospectral, and so is the pair $\left\{A_{H}+\frac{r-k}{n} J, A_{H^{\prime}}+\frac{r-k}{n} J\right\}$ (since they are generalized adjacency matrices of $H$ and $H^{\prime}$, respectively). Therefore $A_{G \backslash H}+\frac{r-k}{n} J$ is cospectral with $A_{G^{\prime} \backslash H^{\prime}}+\frac{r-k}{n} J$. Analogously, it follows that $A_{G \backslash H}+\frac{s-k}{n} J$ is cospectral with $A_{G^{\prime} \backslash H^{\prime}}+\frac{s-k}{n} J$. Hence $G \backslash H$, and $G^{\prime} \backslash H^{\prime}$ are cospectral with respect to two different generalized adjacency matrices. So, by Theorem 2, $G \backslash H$ and $G^{\prime} \backslash H^{\prime}$ are cospectral for the generalized spectrum.

For example take $G=G^{\prime}$ to be the Petersen graph, and take for $H$ and $H^{\prime}$ the two different types of cocliques of size 3 (the neighborhood of a vertex, and three vertices from a coclique of size 4), then $G \backslash H$ and $G \backslash H^{\prime}$ are cospectral for the generalized spectrum. In fact, it is the upper right pair of cospectral graphs of Fig. 7. At first glance one might think that Godsil's observation gives rather special cospectral graphs. However, the opposite is true: 
Proposition 9. Any pair $\left\{K, K^{\prime}\right\}$ of graphs that are cospectral with respect to the generalized spectrum can be constructed from a strongly regular graph, by the method described in Proposition 8 with $G=G^{\prime}$.

Proof. It is known (cf. [39]), that any graph is an induced subgraph of a strongly regular graph. Let $G$ be a strongly regular graph that contains the disjoint union of $K$ and $K^{\prime}$ as an induced subgraph. Then $H=G \backslash K$ and $H^{\prime}=G \backslash K^{\prime}$ are cospectral with cospectral complements, and the method applies.

\subsection{Graphs cospectral with distance-regular graphs}

In [15], several families of distance-regular graphs have been dealt with, but with the exception of one graph, they turned out to be not DS in all cases. These families include the Johnson graphs, the Doubled Odd graphs, the Grassmann graphs, the Doubled Grassmann graphs, the antipodal covers of complete bipartite graphs, and many of the Taylor graphs. Typically, the proofs go in two steps. The first step uses Godsil-McKay switching or the partial-linear-space technique (as described in [13], Sectons 3.2 and 3.3) to find a cospectral mate, and in the second step it is shown that the graph is not distance-regular anymore and therefore non-isomorphic to the original graph. One important exception to this approach is the discovery by Van Dam and Koolen [16] of cospectral graphs for the Grassmann graphs $J_{q}(2 d+1, d)(q>1$ prime power, and $d \geq 2)$. For these graphs the second step did not work, because the new graphs turned out to be distance-regular again. Nevertheless, they are non-isomorphic to the original graphs, and thus provide a new infinite family of distance-regular graphs (with unbounded diameter). The paper [15] also contains a table for the number of cospectral graphs for distance-regular graphs on at most 70 vertices, and a proof that the Ivanov-Ivanov-Faradjev graph is DS (which was announced in [13]).

We remark that (by mistake) the antipodal 7-cover of $K_{9}$ is not mentioned in the table in [13] of distance-regular graphs that are DS. Degraer [19] found a new distance-regular antipodal 3-cover of $K_{14}$ and three new distance-regular antipodal 3covers of $K_{17}$, thus showing that these and the original ones are not DS. We think that finding cospectral graphs for distanceregular antipodal covers of complete graphs that are not distance-regular themselves, is one of the interesting problems in this area.

Another interesting and challenging problem is that of the Hamming graphs. Bang and Koolen [2] showed that if $G$ is a cospectral graph of the Hamming graph $H(3, q)$ for $q>3$, and the induced graph on the set of neighbors of a vertex in $G$ is always a disjoint union of three complete graphs, then $G$ is isomorphic to the Hamming graph. Bang et al. [1] proved that for fixed $n$, and $q$ large enough, a cospectral graph of the Hamming graph $H(n, q)$ has the property that the induced graph on the set of neighbors of a vertex is always a disjoint union of $n$ complete graphs $K_{q-1}$. Thus it is proven that $H(3, q)$ is DS, provided $q$ is large enough.

\section{Acknowledgements}

The authors are grateful to Chris Godsil for many discussions on the subject, and to Behruz Tayfey-Rezaie for giving several relevant remarks on a first draft of the paper.

Warning. Because the time between writing this paper and appearing of it in this special issue is unusually long, some very recent developments may not be mentioned, and some of the stated open problems may have been solved.

\section{References}

[1] S. Bang, E.R. van Dam, J.H. Koolen, Spectral characterization of the Hamming graphs, Linear Algebra Appl., doi:10.1016/j.laa.2007.10.026.

[2] S. Bang, J.H. Koolen, Graphs cospectral with $H(3, q)$ which are locally disjoint union of at most three complete graphs, Asian-European J. Math. 1 (2008) $147-156$

[3] R. Boulet, B. Jouve, The Lollipop Graph is determined by its spectrum, Electron. J. Combin. 15 (2008) R74.

[4] A.E. Brouwer, A. Neumaier, The graphs with spectral radius between 2 and $\sqrt{2+\sqrt{5}}$, Linear Algebra Appl. 114/115 (1989) 273-276.

[5] F.C. Bussemaker, W.H. Haemers, E. Spence, The search for pseudo orthogonal Latin squares of order six, Des. Codes Cryptogr. 21 (2000) 77-82.

[6] P.J. Cameron, Random strongly regular graphs? Discrete Math. 273 (2003) 103-114.

[7] P.J. Cameron, Strongly regular graphs, in: L.W. Beineke, R.J. Wilson (Eds.), Topics in Algebraic Graph Theory, Cambridge Univ. Press, 2004, pp. $203-221$.

[8] A.A. Chesnokov, W.H. Haemers, Regularity and the generalized adjacency spectra of graphs, Linear Algebra Appl. 416 (2006) $1033-1037$.

[9] S. Chowla, P. Erdös, E.G. Straus, On the maximal number of orthogonal Latin squares of a given order, Canad. J. Math. 12 (1960) $204-208$.

[10] K. Coolsaet, The uniqueness of the strongly regular graph srg(105,32,4,12), Bull. Belg. Math. Soc. Simon Stevin 12 (2005) $707-718$.

[11] D.M. Cvetković, M. Doob, H. Sachs, Spectra of Graphs, third ed., Johann Ambrosius Barth Verlag, 1995, Deutscher Verlag der Wissenschaften, first ed. Berlin 1980; Academic Press, New York, 1980.

[12] D.M. Cvetković, P. Rowlinson, S. Simić, Signless Laplacians of finite graphs, Linear Algebra Appl. 423 (2007) 155-171.

[13] E.R. van Dam, W.H. Haemers, Which graphs are determined by their spectrum? Linear Algebra Appl. 373 (2003) $241-272$.

[14] E.R. van Dam, W.H. Haemers, J.H. Koolen, Cospectral graphs and the generalized adjacency matrix, Linear Algebra Appl. 423 (2007) $33-41$.

[15] E.R. van Dam, W.H. Haemers, J.H. Koolen, E. Spence, Characterizing distance-regularity of graphs by the spectrum, J. Combin. Theory A 113 (2006) $1805-1820$.

[16] E.R. van Dam, J.H. Koolen, A new family of distance-regular graphs with unbounded diameter, Invent. Math. 162 (2005) 189-193.

[17] E.R. van Dam, E. Spence, Combinatorial designs with two singular values. I. Uniform multiplicative designs, J. Combin. Theory. A 107 (2004) $127-142$.

[18] E.R. van Dam, E. Spence, Combinatorial designs with two singular values. II. Partial geometric designs, Linear Algebra Appl. 396 (2005) $303-316$.

[19] J. Degraer, Isomorph-free exhaustive generation algorithms for association schemes, Thesis, Ghent University, 2007.

[20] J. Degraer, K. Coolsaet, Classification of some strongly regular subgraphs of the McLaughlin graph, Discrete Math. 308 (2008) 395-400.

[21] D.G. Fon-Der-Flaass, New prolific constructions of strongly regular graphs, Adv. Geom. 2 (2002) 301-306. 
[22] N. Ghareghani, G.R. Omidi, B. Tayfeh-Rezaie, Spectral characterization of graphs with index at most $\sqrt{2+\sqrt{5}}$, Linear Algebra Appl. 420 (2007) 483-489.

[23] C.D. Godsil, B.D. McKay, Constructing cospectral graphs, Aequationes Math. 25 (1982) 257-268.

[24] C.D. Godsil, B.D. McKay, Products of graphs and their spectra, in: L.R.A. Casse, W.D. Wallis (Eds.), Combinatorial Mathematics IV, in: Lecture Notes in Mathematics, vol. 560, Springer, Berlin, 1976, pp. 61-72.

[25] W.H. Haemers, X. Liu, Y. Zhang, Spectral characterizations of lollipop graphs, Linear Algebra Appl. 428 (2008) 2415-2423.

[26] W.H. Haemers, E. Spence, Enumeration of cospectral graphs, European. J. Combin. 25 (2004) 199-211.

[27] E.M. Hagos, Some results on graph spectra, Linear Algebra Appl. 356 (2002) 103-111.

[28] P.L. Hammer, A.K. Kelmans, Laplacian spectra and spanning trees of threshold graphs, Discrete Appl. Math. 65 (1996) $255-273$.

[29] C.R. Johnson, M. Newman, A note on cospectral graphs, J. Combin. Theory. B 28 (1980) 96-103.

[30] M. Lepović, Some results on starlike trees and sunlike graphs, J. Appl. Math. Comput. 11 (2003) 109-123.

[31] M. Lepović, I. Gutman, No starlike trees are cospectral, Discrete Math. 242 (2002) 292-295.

[32] X. Liu, Y. Zhang, X. Gui, The multi-fan graphs are determined by their Laplacian spectra, Discrete Math. 308 (2008) 4267-4271.

[33] M. Muzychuk, A generalization of Wallis-Fon-Der-Flaass construction of strongly regular graphs, J. Algebraic Combin. 25 (2007) 169-187.

[34] A. Neumaier, Strongly regular graphs with smallest eigenvalue $-m$, Arch. Math. 33 (1979) 391-400.

[35] G.R. Omidi, K. Tajbakhsh, Starlike trees are determined by their Laplacian spectrum, Linear Algebra Appl. 422 (2007) 654-658.

[36] X. Shen, Y. Hou, Y. Zhang, Graph $Z_{n}$ and some graphs related to $Z_{n}$ are determined by their spectrum, Linear Algebra Appl. 404 (2005) 58-68.

[37] J.H. Smith, Some properties of the spectrum of a graph, in: R. Guy, et al. (Eds.), Combinatorial Structures and their Applications (Proc. Conf. Calgary, 1969), Gordon and Breach, New York, 1970, pp. 403-406.

[38] D. Stevanović, Research problems from the Aveiro Workshop on Graph Spectra, Linear Algebra Appl. 423 (2007) 172-181.

[39] V.H. Vu, On the embedding of graphs into graphs with few eigenvalues, J. Graph Theory 22 (1996) 137-149.

[40] W. Wang, C.-X.Xu, A sufficient condition for a family of graphs being determined by their generalized spectra, European J. Combin. 27 (2006) $826-840$

[41] W. Wang, C.-X. Xu, On the spectral characterization of T-shape trees, Linear Algebra Appl. 414 (2006) 492-501.

[42] W. Wang, C.-X. Xu, An excluding algorithm for testing whether a family of graphs are determined by their generalized spectra, Linear Algebra Appl. 418 (2006) 62-74.

[43] W. Wang, C.-X. Xu, Note: On the generalized spectral characterization of graphs having an isolated vertex, Linear Algebra Appl. 425 (2007) $210-215$.

[44] R.M. Wilson, An existence theory for pairwise balanced designs, III: Proof of the existence conjectures, J. Combin. Theory A 18 (1975) 71-79.

[45] R. Woo, A. Neumaier, On graphs whose spectral radius is bounded by $\frac{3}{2} \sqrt{2}$, Graphs Combin. 23 (2007) 713-726. 